\title{
Intorno ad alcune forme di numeri primi.
}

\author{
(del prof. A. Gexocchi, a Torino).
}

Provo il celebre Dirichere col mezzo delle serie e di considerazioni infinitesimali che la forma lineare $a x+b$, se $a$ o $b$ sono duc numeri interi primi tra loro, contiene una infinità di numcri primi. Potendo esser utile, specialmente nella dottrina dei numeri, il considerare una medesima questione in diversi aspetti, dimostrerí qui alcuni casi particolari dello stesso teorerna con un metodo piu semplice $c$ anzi elementare che condurrd insieme ad alcune proprieta notabili di corte funzioni.

$1^{0}$ Supposto $k$ un numero intoro e positivo, fanciamo

$$
(a+(b))^{k}=A_{k}+B_{k}(\bar{b},
$$

intendendo con $A_{a}$ e $B_{k}$ clue funzioni intero di $a$ e $b$.

Si arrà nello stesso tempo $(a-\sqrt{b})^{k}=A_{k}-B_{k} \sqrt{b}$, o quindi

$$
A_{k}=\frac{(a-1-\sqrt{6})^{k}+(n-16)^{k}}{2}, \quad B_{k}=\frac{(a+\sqrt{6})^{k}-(a-\sqrt{6})^{k}}{2 \sqrt{b}^{5}} ;
$$

di piì, ponendo

$$
r=a^{2}-b, \quad z=2\left(a^{2}+b\right)
$$

si aurat

$$
\begin{aligned}
& A^{2}{ }_{k}-B I^{2}{ }_{k}=r^{2} \text {, } \\
& 2\left(A^{2}{ }_{k}+b B^{2}{ }_{k}\right)=z^{k}-k r^{2} z^{k-2}+\frac{k(k-3)}{1.2} r^{1} z^{k-1}-\mathrm{crc}
\end{aligned}
$$

Simo a $0 b$ duc numeri interi; saranno tali ancho $A_{k}$ o $B_{k}$; c se $A_{k}$ e $b B_{k}$ hamno un fattor primo comune, esso per la (4) saris divisore anche di $r$, e quindi per la (J) saril parimente divisore di $z$; dungue un tal fittore sara 
2 oppure dividerà insieme $a^{2}+b$ e $a^{2}-b$, per ciò anche $2 a^{2}$ e $2 b$, ossia $a$ e $b$. Adunque se $a$ e $b$ sono primi fra loro, e l'uno pari, e l'altro impari, anche $A_{z}$ e $b B_{k}$ saramo primi tra loro, e l'uno pari. I'altro impari a causa di $r$ impari e della equazione (4). Se $a$ e $b$ sono ambedue impari, $A_{k}$ e $B_{k}$ saranno pari poichè sono somme di termini della forma ma $a^{p} b^{q}$, che è pari 0 impari come $m$, onde si potrà ad un tal termine sostituire $m$, ovvero supporre $a=b=1$, il che darà $A_{k}=B_{k}=2^{k-1}$ valori pari. Ma non avrauno comuue alcun fattore impari se $a$ e $b$ non ne hanno: noi supporremo sempre $a \quad c \quad b$ primi fra loro.

2. Se $n$ è un numero intero e positivo si ha

$$
\begin{gathered}
\frac{l^{n}-u^{n}}{t-u}=l^{n-1}+t^{n-2} u+t^{n-3} u^{2}+\cdots+t u^{n-2}+u^{n-1} \\
=n u^{n-1}+(t-u)\left(\frac{l^{n-1}-u^{n-1}}{t-u}+u \frac{t^{n-2}-u^{n-2}}{t-u}+u^{2} \frac{l^{n-3}-u^{n-3}}{t-u}+\cdots+u^{n-2}\right) \\
=n u^{n-1}+(t-u)\left(l^{n-2}+2 t^{n-3} u+3 t^{n-4} u^{2}+\cdots+(n-2) t u^{n-3}+(n-1) n^{n-2}\right):
\end{gathered}
$$

scambiando $t$ con $u$ e poi sommando le due equazioni si otterrà

$$
\begin{gathered}
\frac{t^{n}-u^{n}}{t-u}=n \cdot \frac{b^{n-1}+u^{n-1}}{2}-V(t-u), \\
V=(n-2) \frac{t^{n-2}-t^{n-2}}{2}+(n-4) t u^{t^{3-4}-u^{n-4}} \\
2
\end{gathered}
$$

Sia $n$ un altro numero intero e positivo, e si faccia $t=(a+\sqrt{b})^{n+b}, u=(a-\sqrt{b})^{m ;}$; ne risulterà $t u=\left(a^{2}-b\right)^{m}, l-u=2 \sqrt{b} . B_{m}, t^{n}-u^{n}=2 \sqrt{b} . B_{m n}, l^{k}-u^{k}=2 \sqrt{b} \cdot B_{k m}$, $t^{n-1}+u t^{n-1}=2 \Lambda_{a n-n}$, ritenute lo supposizioni del numero precedente; quindi

$$
\frac{B_{m n}}{B_{m}}=n A_{m n-m}-2 V \sqrt{b} . B_{m},
$$

- $V$ prenderà la forma $I I \sqrt{b}$ con $H$ numero intero, onde anche il quoziente $\frac{B_{m n}}{B_{m}}$ sarà un numero intero, ossia $B_{u n n}$ sarà divisibile per $B_{m}$. Per conseguenza anche $B_{m n-m}$ sard divisibile pel $B_{m}$. Ma secondo la stessa eguaglianza il numero $\frac{B_{n}}{B_{n}}$ non può aver commi con $B_{n n}$ altri divisori che quelli di $n A_{m n-m}$ : e d'altra parte $B_{m n-m}$ cho deve contenerc tutti i divisori di $B_{m}$ non puó, pel n." precerlente, aver comuno con $A_{u n-m}$ alcun divisore impari, cosicche 
nessun divisore impari di $B_{m}$ sarà comune ad $A_{m n-m}$ : dunque $\frac{B_{m n}}{B_{m}}$ non potrà aver comuni con $B_{m}$ altri divisori impari che quelli dell'esponente $n$.

3. Sia $p$ un divisore impari del numero intero $B_{k}$ nel caso di $k=m$, e sia $h$ il più piccolo valore di $k$ che rende $B_{k}$ divisibile per $p$ : dico che $h$ sarà un divisore di $m$. Imperocchè se $h$ non eguaglia $m$ sarà minore di $m$ e se non divide $m$ esattamente, sia $q$ il quoziente e $h^{\prime}$ il resto della divisione, onde $m=h q+h^{\prime}$ : posto $B_{n k}=M p, B_{h}:=H p$, sarà eziandio $B_{h q}=H^{\prime} p$ con $M, H, H^{\prime}$ interi poichè $B_{k, t}$ sarà divisibile per $B_{h}\left(\mathrm{n}^{\circ} 2^{\circ}\right)$, e ne dedurremo

$$
\left(\frac{a+\sqrt{b}}{a-\sqrt{b}}\right)^{m}-1=\frac{2 \sqrt{b}}{(a-\sqrt{b})^{m}} \cdot M p,\left(\frac{a+\sqrt{b}}{a-\sqrt{b}}\right)^{h q}-1=\frac{2 \sqrt{b}}{(a-\sqrt{b})^{h q}} \cdot H^{\prime} p
$$

e però

$$
\left(\frac{a+\sqrt{b}}{a-\sqrt{b}}\right)^{h_{2}}\left[\left(\frac{a+\sqrt{b}}{a-\sqrt{b}}\right)^{h^{\prime}}-1\right]=\frac{2 p \sqrt{b}}{(a-\sqrt{b})^{h_{g}}}\left[\frac{M}{(a-\sqrt{b})^{n^{\prime}}}-H^{\prime}\right]
$$

ossia

$$
\left(a^{2}-b\right)^{h^{\prime}} . B_{\iota^{\prime}}=p\left[M(a-\sqrt{b})^{k q}-H^{\prime}(a-\sqrt{b})^{m}\right],
$$

dal cui secondo membro dovendo sparire $\sqrt{6}$ come sparisce dal primo, risultorà che $p$ è un divisore del primo membro. Ma se un divisor primo di $p$ dividesse $\left(a^{2}-b\right)^{h_{q}}$ dividerebbe $a^{2}-b$ e quindi dividendo insieme $r$ e $B_{m}$ dividerebbe anche $A_{m}$ per la (b), mentre $A_{m}$ e $B_{m}$ non hanno comunc alcun fattore impari: dunque $p$ sarebbe divisore di $B_{h^{\prime}}$ \& $h^{\prime}$ sarebbe $<h$, la qual cosa è contraria all'ipotesi.

Segue da ciò che se $B_{k}$ is multiplo di $p$ per due ralori $k=m$ e $k=m^{\prime}$ dell'indice, saranno $m$ e $m^{\prime}$ multipli di $h$; quindi anclie il massimo comun divisore $\mu$ di $m$ c $m^{\prime}$ sarà multiplo di $h$, c in conseguenza sarà multiplo di $p$ anche $B_{\mu}$.

4. ${ }^{\circ}$ Secondo teoremi noti, chiamato $p$ un numero primo impari, se $b \dot{\theta}$ un residuo quadratico di $p$, il numero $B_{p_{-1}}$ è divisibile per $p$, c se $b$ ì un non residuo quadiatico di $p$, il numero $B_{p+1}$ e divisibile per $\hat{\imath}$ : cio qualunque numero intero sia $a\left(^{*}\right)$. Sia pertanto $\frac{B_{a, a}}{B_{a, 2}}=P$, e $p$ un divisor primo impari di

() Mém. Aoad. de Borlin, 1773, pag. 332; Gauss, Distulis. Arithm. art. 123. 
$P$ che non divida $n$ : sarà $p$ un divisore di $B_{m n}$ che non dividerà $B_{n}\left(\mathrm{n} .^{0} 2^{0}\right)$, c nol primo caso $p-1$, nel secondo $p+1$ avà comune coll' indice mn un divisole che sarà estraneo all'indice $m$. Se dunque $n$ sia un numero primo e $m$ una sua potenza $n^{i-1}$, dovrà $p \pm 1$ aver per divisore $m n=n n^{i}$, onde sarà $p \mp 1=n^{i} z, p=n^{i} z \pm 1$. E cosi nel primo caso avremo trovato un numero primo delia forma $n^{i} z+1$, nel secondo un uumero primo della forma $n^{i} z-1$.

Nel caso di $i=1$, sarà $B_{n}=B_{1}=1, B_{m n}=B_{n}, P=B_{n}$. Il numero $n$ può anche essere $=2$.

$5 .^{\circ}$ Se $k$ è impari, avromo dalle (2)

$$
\left.\begin{array}{l}
A_{k}=a^{k}+\frac{k(k-1)}{1.2} a^{k-1} b+\cdots+k a b^{\frac{k-1}{2}} \\
B_{k}=k a^{k-1}+\frac{k(k-1)(k-2)}{1.2 .3} a^{k-3} b+\cdots+\frac{k(k-1)}{1.2} a^{2} b^{\frac{k-3}{2}}+b^{\frac{k-1}{2}}:
\end{array}\right\}
$$

dalla second a delle quali è chiaro che se $b$ è primo ad $a$, e $a$ è multiplo di $n$, sarà $B_{k}$ primo ad $\hat{n}$. Quindi preso $a$ multiplo di $n$, fatto $b=1$, e chiamato $p$ un divisor primo impari di $P$ come nel $n .^{\circ}$ precedonte, avremo $p=n^{i} z+1$, poichè $b$ sarà residuo quadratico di $p$, e $p$ non sarà divisore di $n$, essendo $B_{m n}$ e perciò $P$ primo ad $n$.

Ciò vale per $n$ numero primo impari e anche per $n=2$.

Supponendo invece $b=-1$, a pari e multiplo di $n$, potremo trovare un numero primo $p$ della forma $n^{i} z-1$, quando $n$ sia della forma $4 K+3$. Poichè i numeri $m$ e $m n$ saramo l'uno della forma $4 K+1$ e l'altro della forma $4 K+3$, ed essendo $B_{k}=C a^{2}+(-1)^{\frac{k-1}{2}}$, anche i numeri $B_{m}$ e $B_{m i n}$ saranno l'uno della forma $4 K+1$ e l'altro della forma $4 K+3$, onde il quoziente $\frac{B_{a n}}{B_{n}}$ ossia $P$ sarà della forma $4 K+3$ e avrà qualche divisore primo $p$ della stessa forma: questo numero primo essendo diverso da $n$ e avendo -1 per non residuo quadratico sarà $=n^{i} z-1$.

6. Supponiamo anche $b= \pm 2$. Se $a$ e $k$ sono impari, abbiamo, separando i multipli di 8 ,

$$
B_{k}=k \pm \frac{1(k-1)(k-2)}{2.3} \cdot 2 a+\frac{k(k-1)(k-2)(k-3)(k-4)}{2.3 .4 .3} \cdot 4+8 G,
$$

almeno per $k>6$. Ne segue che quando $k$ abbia la forma $8 K+1$, anche 
$B_{k}$ sarà della stessa forma. Se $k$ è della forma $8 K-1$, avremo $B_{k}=-1 \pm\left(\frac{-1 \cdot-2 .-3}{3}\right) a+\left(\frac{-1 \cdot-2 \cdot-3 \cdot-4.5}{2.3 .4 .5}\right) \cdot 4+8 C^{\prime}=8 C^{\prime \prime} \pm 2 a-5$, e quindi $B_{k}$ sarà della forma $8 K-3$, se nel caso di $b=2$ prenderemo $a$ della forma $4 K-1$, e nel caso di $b=-2$ prenderemo a della forma $4 K+1$. Supposto $n$ della forma $8 K-1$, e $n=n^{i-1}$, uno dei numeri $m$ e $m n$ avrà la forma $8 K+1$ e l'altro la forma $8 K-1$; per cio uno dei numeri $B_{n}$ e $B_{m n}$ avrà la forma $8 K+1$, e l'altro la forma $8 K-3$, onde il quoziente $P$ sarà della forma $8 K-3$, e avrà almeno un divisore primo $p$ d'una delle forme $8 K \pm 3$, che sarà diverso da $n$ essendo $n$ della forma. $8 K-1$. Nel caso di $b=2$, sarà $b$ non residuo di $p$, e ne risulterà $p=n^{i} z-1$. Sarà. lo stesso nel caso di $b=-2$, se $p$ ha la forma $8 K-3$; se invece $p$ ha la forma $8 K+3$, sarà $b$ residuo di $p$ e si avrà $p=n^{i} z+1$, ma in questo caso $P$ ammetterả eziandio un divisor primo della forma $8 K-1$, di cui $b$ sarà non residuo e che sarà diverso da $n$ se prendasi a multiplo di $n$, e chiamandolo $p$ si avrà ancora $p=n^{i} z-1$.

Se $k$ è della forma $8 K+3$, avremo $B_{k}=3 \pm 2 a+8 C^{\prime}$, che sarà della forma $8 K-3$ se nel caso di $b=2$ prenderemo a della forma $4 K+1$, e nel caso di $b=-2$ prenderemo a della forma $4 K-1$. Se infine $k$ è della forma $8 K-3$, avremo $B_{k}=-3 \mp 4.5 a-5.4+8 C^{\prime}$, che per $a$ impari è semple della stessa forma $8 K-3$. Quindi supposto $n$ d' una delle forme $8 K \pm 3$, i numeri $m$ e $m n$ saranno l'uno della forma di $n$, e l'altro della forma $8 K+1$; $B_{m}$ e $B_{t, n}$ saranno l'uno della forma $8 K+1$, l'altro della forma $8 K-3$, onde (preso a multiplo di $n$ ) si giungerà ancora alle medesime conclusioni di poc' anzi.

Convien notare che anche quando facciamo $b$ negativo noi supponiamo positivi i valori di $B_{k}$ perchè prendendo a abbastanza grande si puỏ sempre rendere il primo termine $k a^{k-1}$ del polinomio $B_{k}$ superiore al complesso di tutti gli altri termini.

7. Le precedenti proposizioni possono servire ad una dimostrazione del teorema di Fermat sulla possibilità di risolvere con numeri interi l'equaziono

$$
2 n=x^{2}+y^{2}+z^{2}
$$

ogniqualvolta $n$ sia un numero primo $8 K-1$. Imperoccho abbiamo provato che o possibile trovar un numero primo $8 K \pm 3$ compreso nella formola $p=n z-1:$ dovendo $z$ esser pari, faremo $z=2 s$, e avremo $1+p=2 n s$, 
donde coi simboli di LEGENDrE trarremo $\left(\frac{-p}{n}\right)=1$, e quindi per legge di reciprocazione trarremo $\left(\frac{n}{p}\right)=-1$ nel caso di $p=8 \Pi-3$, e $\left(\frac{n}{p}\right)=+1$ nel caso di $p=8 K+3$; inoltre nel primo caso è $\left(\frac{-2}{p}\right)=-1$, e nel secondo $\left(\frac{-2}{p}\right)=+1:$ dunque in ambedue i casi $\left(\frac{-2 n}{p}\right)=1$, sicchè poträ rendersi divisibile per $p$ la formola $1+2 n t^{2}$, e chiamato $r$ il quoziente si arrà $1+2 n t^{2}=p r=r(2 n s-1)$, ossia $2 n t^{2}+r-2 n r s=-1$.

Potendosi, dato $n$, determinare valori interi di $r, s, t$, che soddisfacciano a questa equazione, cio basta, come avverti il Dirichlet (*), per dedurne che $2 n$ si può trasformare nell' espressione ternaria $x^{2}+y^{2}+z^{2}$.

8. Sia $n$ un numero primo impari qualsivoglia, $b$ uno de'suoi non residui quadratici: si avrà $b^{\frac{n-1}{2}} \equiv-1(\bmod . n)$, e quindi $B_{n}=C n-1$ per la seconda delle $(6)$ : dunque $B_{n}$ non sarà divisibile per $n$ e avrà qualche divisore primo di cui $b$ sarà non residuo, poichè ogni divisore primo di $B_{n}$ del quale $b$ sia residuo quadratico deve avere la forma $n z+1\left(n .^{\circ} 4^{\circ}\right)$ e quindi se tutti i divisori primi di $B_{n}$ avessero $b$ per residuo quadratico sarebbero tutti della forma $n z+1$ e $B_{n}$ loro prodotto avrebbe pure la stessa forma. Chiamato dunque $p$ un divisore primo di $B_{n}$ avente $b$ per non residuo quadratico, sarà $p=n z-1$; e così viene dimostrata l' esistenza di numeri primí di questa forma che inoltre debbano avere per non residuo il numero $b$ scelto ad arbitrio fra $i$ non residui di $n$.

9. Si ha dalla (1)

$$
A_{m n}+B_{m n} \sqrt{b}=(a+\sqrt{b})^{m n}=\left(A_{m}+B_{m} \sqrt{b}\right)^{n}
$$

e quindi se $n$ è impari

$$
\begin{aligned}
& A_{m n}=A_{m}{ }^{n}+\frac{n(n-1)}{1 \cdot \mathscr{2}} A_{m}{ }^{n-2} B_{m}{ }^{2} b+\cdots+n A_{m} B_{m}{ }^{n-1} b^{\frac{n-1}{2}}, \\
& B_{m n}=n A_{m}{ }^{n-1}+\cdots+\frac{n(n-1)}{1 \cdot \underline{y}} A_{m}{ }^{2} B_{m}{ }^{n-2} b^{\frac{n-3}{2}}+B_{m}{ }^{n} b^{\frac{n-1}{2}}:
\end{aligned}
$$

(v) G. Crelle tom. XI, pag. 228. 
donde supposto $n$ un numero primo, risulta

$$
A_{m n} \equiv A_{n}^{n} \equiv A_{m n}, \quad B_{m n} \equiv B_{m}^{n} \eta^{\frac{n-1}{2}} \equiv B_{m}\left(\frac{l}{n}\right) \quad(\bmod . n) \text {. }
$$

Se $b$ è non residuo quadratico di $n$, sarà dunque $B_{m n} \equiv-B_{m}(\bmod . n)$; e poichè abbiam già trovato $B_{n} \equiv-1$, facendo successivamente $m=n, n^{2}, n^{3}, \ldots$ ne trarremo di mano in mano $B_{i n} \equiv-B_{n} \equiv 1, B_{n n n} \equiv-B_{n n} \equiv-1$, ecc. talchè supposto $m=n^{i-1}$, avremo sempre $B_{m n} \equiv-B_{m} \equiv \pm 1(\bmod , n)$, e dei due numeri $B_{m}, B_{m i}$ l'uno drrà la forma $C n+1$, l'altro arrà la forma $C n-1$. Pertanto il quoziente $P$ avrà sempre la forma $C n-1$, non sarà divisibile per $n$, e dovrà avere qualche divisor primo $p$ di cui $b$ sarà non residuo, essendo della forma $n z+1$ tutti i divisori primi di $B_{n n}$ de'quali $b$ è residuo quadratico $\left(n .^{\circ} 4^{\circ}\right)$ e della stessa forma tutti i prodotti formati con questi. Arrem dunque $p=n^{\imath} z-1$; e sarà così dimostrata l'esistenza di sifiatti numeri primi $p$, aggiunta la condizione che $b$ sia non residuo quadratico tanto di $n$ quanto di $p$.

10. Abbiamo

$$
\begin{aligned}
& A_{2}+B_{2} \sqrt{b}=(a+\sqrt{b})^{2} \\
& A_{2 m}+B_{2 m} \sqrt{b}=(a+\sqrt{b})^{2 m}=\left(A_{m}+B_{m} \sqrt{b}\right)^{2}
\end{aligned}
$$

donde

$$
A_{2}=a^{2}+b, \quad B_{2}=2 a, \quad A_{2^{m}}=A_{m}{ }^{2}+b B_{n}^{2} ; \quad B_{a^{m}}=2 A_{m} B_{m} .
$$

Quindi $b_{2}, B_{1}, B_{6}, \ldots$ sono tutti numeri jari.

Preso $a=2, b=-1$, risulter'à $A_{2}=3, B_{2}=4, A_{2,2}=A_{m}{ }^{2}-B_{n n}{ }^{2}$ : saranno numeri impari $A_{4}, A_{8}, A_{16}, \ldots$; di più l'equazione $B_{2 n}=2 A_{n 2} B_{n i}$ darà $B_{4}$ multiplo di $8, B_{s}$ multiplo di 16 , ecc. Si trovera $1_{4}$ negativo, $A_{8}$ negativo, $A_{16}$ positivo. Sia greneralmento $A_{2 m}$ positivo, cioe $A_{\mathrm{in}}{ }^{2}>B_{A^{2}}$, e facciamo $A_{m}=k B_{m}$, onde $k^{2}>1:$ avremo $A_{\mathrm{g} n}=\left(k^{2}-1\right) B_{m}{ }^{2}, B_{2 m}=2 k B_{m}{ }^{2}$, e fatto $A_{2 m}=k^{\prime} B_{m g}$, ne trarremo $k^{\prime}=\frac{1}{2} k-\frac{1}{2 k}$, talchè $k^{\prime}$ sarà positivo se $k$ è positivo, negativo se $k$ i negativo, ma in entrambi i casi sarà quanta al valor assoluto $l^{\prime}<\frac{1}{z} h_{2}$. Similmente fatto $A_{t, 2,4}=k^{\prime \prime} J_{1: n}$, si avrà in valor assoluto $k^{\prime \prime}<\frac{1}{2} k^{\prime}$, o però $k^{\prime \prime}<\frac{1}{4} k$; fatto $A_{S_{m}}=k^{\prime \prime \prime} 3_{;}$; , si avrà $k^{\prime \prime \prime}<\frac{1}{2} k^{\prime \prime}<\frac{1}{8} k$, e così in progresso finche i coefficienti $k, k^{\prime}, k^{\prime \prime}, .$, resteranno numericamente superiori all' unita. II a continuando la serio $k, \frac{1}{2} k, \frac{1}{4} k, \frac{1}{8} k, \ldots$ si troverà infine un termine numericamente minore di 1 ; dunque un tal termine si troverả 
anche nella serie $k, k^{\prime}, k^{\prime \prime}, \ldots$, e supponendo che sia $k^{(i)}$, fatto $m^{\prime}=2^{i} m$, si avrà numericamente $A_{i t^{\prime}}<B_{n^{\prime}}$, e sarà negativo $A_{2 m^{\prime}}$. Adunque nella scrie $A_{2}, A_{4}, A_{8}, \ldots$ si troveranno termini negativi il cui indice sia maggiore di qualsiasi numero dato.

Di qui si deduce l'esistenza di numeri primi della forma $2^{i} z-1$, essendo $i$ un numero dato qualsivoglia. Poichè indicata con $m$ una potenza di 2 eguale o superiore a $2^{i-1}$ tale che $A_{n}$ sia negativo, si avrà il quozionte $P=\frac{B_{2 m}}{B_{n}}=2 . A_{m}$, che sarà negativo; ma fatto $m=2 m^{\prime}$, si avrà $-A_{m}=B_{m n^{\prime}}{ }^{2}-A_{m^{\prime}}{ }^{2}$, e saranno $A_{m^{\prime}}$ impari, $B_{m^{\prime}}$ divisibile almeno per 4 , onde $-A_{n n}$ sarà un numero positivo della forma $8 K-1$, e $-P$ un numero positivo della forma $2(8 K-1)$ : dunque $P$ ammetterà per divisore qualche numero primo della forma $4 K+3$, e chiamatolo $p$ si avrà $p=2 m z-1\left(n .{ }^{\circ} 4^{0}\right)$, essendo $b=-1$ non residuo di $p$. Il numero $p$ avrà la forma voluta perchè $2 m$ sarà eguale 0 multiplo di $2^{i}$. Cosi tali Inumeri primi si troveranno fra i divisori della formola

$$
\frac{(2+\sqrt{-1})^{k}-(2-\sqrt{-1})^{k}}{2 \sqrt{-1}}
$$

supponendo $k$ una potenza del 2 .

11. Consideriamo finalmente un numero $m$ composto di fattori primi differenti, e rappresentiamo con $n_{1}$, uno qualsiasi di tali fattori, con $n_{2}, n_{3}, n_{4}, \ldots$ uno qualsiasi dei loro prodotti a due a due, a tre a tre, a quattro a quattro, ccc.; poniamo inoltre $\frac{m}{n_{1}}=m_{1}, \frac{n}{n_{2}}=m_{2}, \frac{m}{n_{3}}=m_{3}$, ecc. Si faccia $b=1$, e si prenda a multiplo di tutti i fattori primi $n_{1}$. Sarà $B_{m}$ multiplo di tutti i valori di $B_{m_{1}}$ compondenti ai diversi valori di $n_{1}$, e chiamato $M$ il minimo multiplo comune di tali valori di $B_{m_{1}}$ sarà $\frac{B_{j i}}{M}$ un numero intero che indicheremo con $P$ : sarà $P$ come $B_{m}$ primo ad̉ $a$ e perciò a tutti i numeri $n_{1}$; e $\frac{B_{i a}}{B_{i n}}$ che è multiplo di $P$ non potendo aver comune con $B_{n n_{t}}$ altro divisore impari che $n_{1}\left(\right.$ n. $\left.^{\circ} 2^{0}\right)$ poichỏ $m=m_{1} n_{1}$, non avrà comune con $B_{u a_{1}}$ alcun divisore impari. Sia $p$ un divisore primo impari di $P$ : sara $p$ un divisore di $B_{n}$ che non dividerà $B_{m}$ e quindi non dividerà $B_{k}$ qualunque sia $k$ dei divisori di $m$, escluso $m$, poichè $k$ sara multiplo d'alcuno dei valori di $m_{1}$. Ma essendo $b=1$ residuo di $p$, anche $b_{p-1}$ sarà multiplo di $p$ : dunque $p-1$ sarà multiplo di $m\left(n .^{\circ} 3^{n}\right)$ c si avrà $p=m \Sigma+1$. 
Adunque per ogni numero $m$, pari o impari, primo o composto, è dimostrata l'esistenza di numeri primi della forma $m z+1$. Occorrono altri principj pei numeri primi della forma $m z-1$, se $m$ è un numero composto di fattori primi differenti.

12. Dinotiamo generalmente con $f(x)^{k}$ la frazione $\frac{x^{k}-1}{x-1}$, e ritenuto il significato di $m, m_{i}, n_{i}$ dato nel $n .^{\circ}$ precedente poniamo

$$
X=f(x)^{m} \cdot \frac{\Pi \int(x)^{m_{2}} \cdot \Pi f(x)^{n_{4}} \cdot \Pi f(x)^{n_{6}} \ldots}{\Pi f(x)^{m_{4}} \cdot \Pi f(x)^{m_{3}} \cdot \Pi f(x)^{m_{5}} \ldots} .
$$

ove i segni $\Pi$ indicano moltiplicazioni stese a tutti i valori di $m_{1}, m_{2}$, ecc.: sarà $X=0$ l'equazione avente per radici le radici primitive dell'equazione $x^{m}-1=0$. Ora presi $a$ e $b$ primi fra loro, formate le quantità $B_{m_{i}}$, chiamato $M$ il minimo multiplo comune di tali quantità, e fatto $\frac{B_{m}}{M}=P$; potremo dedurre $P$ da $X$ ponendo $x=\frac{a+\sqrt{b}}{a-\sqrt{b}} \cdot$ Poichè avremo

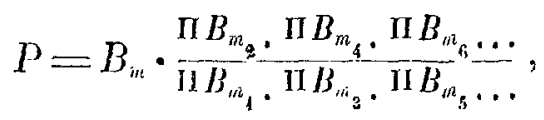

e insieme $f(x)^{k}=\frac{B_{l}}{(n-\sqrt{b})^{h-1}}$, c quindi

$$
X=\frac{p}{(a-\sqrt{b})^{2}}
$$

per un conveniente valore di $\lambda$ formato dalla somma algebrica di tutti i valori di $k-1$. Distinguendo in $\lambda$ la parte derivante dal termine $k$ o la parte derivante dal termine -1 , e chiamando $s$ il numero dei fattori primi diversi di $m$, avremo per la seconda parte

$$
-\left[1-\frac{s}{1}+\frac{s(s-1)}{1.2}-\frac{s(s-1)(s-2)}{1.2 .3}+\cdots\right]=-(1-1)^{s}=0
$$

e la prima parte sarà cspressa da

$$
\begin{gathered}
m-\mathbf{\Sigma} m_{1}+\mathbf{\Sigma} m_{2}-\mathbf{\Sigma} m_{3}+\cdots=m-\mathbf{y} \frac{m}{n_{1}}+\mathbf{\Sigma} \frac{m}{n_{2}}-\mathbf{\Sigma} \frac{m}{n_{3}}+\cdots \\
=m \Pi\left(1-\frac{1}{n_{1}}\right),
\end{gathered}
$$


numero che dinota quanti sono i numeri inferiori ad $m$ e primi ad $m$. Adunque $\lambda$ si ridurà a questo numero; e tale essendo pure il grado del polinomio $X$ rispetto ad $x$, basterà sostituire in questo polinomio il valore di $x=\frac{a+\sqrt{b}}{a-\sqrt{b}}$, e fare sparire i denominatori: allora $X$ sarì trasformato in $P$.

Si supponga $b=-h^{2}$, indicato con $h$ un numero intero; e sia $\rho$ il modulo, $\phi$ l'argomento dell'espressione immaginaria $a+h \sqrt{-1}$, onde $a \pm h \sqrt{-1}=\rho e^{ \pm \Phi} \sqrt{-1}$, e $x=\frac{a+\sqrt{b}}{a-\sqrt{b}}=e^{2 \varphi \sqrt{-1}}$. Rappresentando inoltre con $u$ uno qualsivoglia degl'interi inferiori e primi ad $m$, e stendendo la moltiplicazione a tutti i valori di $u$, potremo scrivere

$$
X=\Pi\left(x-e^{-\frac{2 u \pi}{m} \sqrt{-1}}\right):
$$

ma sarà

$$
x-e^{-\frac{2 u \pi}{m} \sqrt{-1}}=2 \sqrt{-1} \cdot e^{\left(\varphi-\frac{u \pi}{m}\right) \sqrt{-1}} \operatorname{sen}\left(\phi+\frac{u \pi}{m}\right) ;
$$

quindi

$$
X=2^{\lambda}(\sqrt{-1})^{\lambda} \cdot e^{\left(\lambda \varphi-\frac{\pi \Sigma u}{m}\right) l \overline{=1}} \cdot \Pi \operatorname{sen}\left(\phi+\frac{u \pi}{m}\right),
$$

che si riduce a

$$
X=2^{\lambda} e^{\lambda \varphi \sqrt{-1}} \cdot \Pi \operatorname{sen}\left(\phi+\frac{u \pi}{m}\right)
$$

perchè

$$
\sum u=\frac{1}{2} \lambda m, e^{-\frac{1}{2} \lambda \pi \sqrt{-1}}=(-\sqrt{-1})^{\lambda},(\sqrt{-1})^{\lambda}(-\sqrt{-1})^{\lambda}=1
$$

Essendo

$$
P=X(a-V b)^{\lambda}=X \cdot \rho^{\lambda} e^{-\lambda \varphi \sqrt{-1}}
$$

ne risulta

$$
P=2^{\lambda} \rho^{\lambda} . \Pi \operatorname{sen}\left(\phi+\frac{u \pi}{m}\right)
$$

11 maggior valore di $u$ sarà $m-1$, c quindi se l'arco $\phi$ sia maggiore di $\frac{\pi}{m}$ e minore dj $\frac{2 \pi}{m}$, l'arco $\phi+\frac{m-1}{m} \pi$ sarà compreso tra $\pi$ e $2 \pi$ e avrà Annali di Halematica, tomo II. 
un seno negativo, mentre tutti gli altri archi $\phi+\frac{u \pi}{m}$ saranno minori di $\pi$ e avranno seni positivi, il che darà $P$ negativo. Ma avendosi tang $\phi=\frac{h}{a}$, infiniti valori razionali e irrazionali di $a$ e $h$ soddisfaranno alla condizione $\frac{\pi}{m}<\phi<\frac{2 \pi}{m}$, cosicchè in infiniti modi, anche supposti $a$ e $h$ interi, si potrà render $P$ negativo.

13. ${ }^{\circ}$ Sia $m$ impari, e si prenda $h$ impari, a multiplo di 4 e di tutti $i$ fattori primi di $m$. Saranno impari tutti i valori di $m_{1}, m_{2}$, ecc., e così nella espressione (8) tutti gl'indici saranno impari. Ma dalle (6) avremo $B_{k} \equiv b^{\frac{k-1}{2}}$ (mod. 8), e quindi dalla (8) dedurremo $P \equiv b^{\frac{2}{2}} \equiv\left(-h^{2}\right)^{\frac{\lambda}{2}}$ (mod. 8): ora entrando in $m$ almeno due fattori primi differenti il numero $\lambda$ sarà divisibile per 4; dunque sarà $P \equiv h^{\hbar} \equiv 1$ (mod. 8). Supposto inoltre $P$ negativo, ne segue che $-P$ sarà un numero intero positivo della forma $8 K-1$, e arrà qualche divisor primo $p$ della forma $4 K+3$. Sarà $B_{n}$ primo ad $m$ e a $b$ ossia ad $h$; quindi $p$ non sarà divisore di in nè di $h$, ed essendo della forma $4 K+3$, sarả $b=-h^{2}$ uno de'suoi non residui, onde $P$ sarà divisore di $B_{p+1}$. Da ciò ragionando come al $n .^{\circ} 11^{\circ}$ si concluderà che $p+1$ sarà divisibile per $m$, $e$ così $p=m z-1$, e il numero $z$ sarà multiplo di 4 perchè $p$ ha la forma $4 K+3$.

14. ${ }^{\circ}$ Se $m$ è pari ma divisibile soltanto per 2 ovvero per 4 , basta ancora la data dimostrazione, poiche essendo $z$ multiplo di 4 , la forma $m z-1$, dimostrata nel caso di $m$ impari, comprende le due $2 m z^{\prime}-1$ e $4 m z^{\prime \prime}-1$. Per gli altri casi, intenderemo che $n_{1}, n_{2}, n_{3}, \ldots$ rappresentino i fattori primi impari di $m$ e i loro prodotti, e ritenuto $\frac{m}{n_{1}}=m_{1}, \frac{m}{n_{2}}=m_{2}, \frac{m}{n_{3}}=m_{3}, \ldots$ doviemo nella formola (8) per tener conto del fattore 2 sostituire a $\Pi B_{m_{i}}$ il prodotto $B_{\frac{1}{2} n} . \Pi B_{m_{1}}$, e generalmente a $\Pi B_{n_{i}}$ il prodotto $\Pi B_{\frac{1}{2} m_{i \cdot 1}} . \Pi B_{n_{i}}$. Ma si ha $\left(\right.$ n. $\left.{ }^{\circ} 10^{\circ}\right) B_{2 l i}=2 A_{k} B_{k}$, onde $B_{k}=\frac{B_{2 r}}{2 A_{k}}$ : fatte queste sostituzioni, e ommesso il fattor 2 che avrebbe per esponente

$$
1-\frac{s-1}{2}+\frac{(s-1)(s-2)}{1.2}-\cdots=(1-1)^{s-1}=0,
$$


si otterra

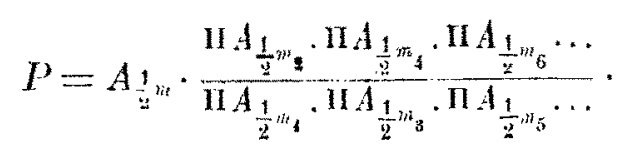

Ora $A_{k}=a^{k}+\frac{k(k-1)}{1.2} a^{k-1} b+$ ecc.; quindi prendendo a impari, h multiplo di 4 , e perỏ $b$ di 16 , arremo $A_{k} \equiv a^{2}(\bmod .16)$. Adunque $p \equiv a^{\frac{1}{2} /}(\bmod .16)$, essendo $\lambda=m-\mathbf{\Sigma} m_{1}+\mathbf{\Sigma} m_{2}-\cdots ;$ ma supponendosi $m$ divisibilo per 8 . $c$ per qualche fattore impari, sari $\lambda$ multiplo di 16 , e $\frac{1}{9} \lambda$ di 8 ; dunque $P \equiv 1$ (mod. 10). Portanto se $P$ i negativo sara $-P$ un numcro positivo della forme $16 K-1$, e arrà qualche divisor primo $p$ della forma $4 K+3$, che sarà diverso da tutti i ralori di $n_{1}$, se si prenderà a multiplo di tutti questi, e che avrà $b$ per non residuo quadratico. Dal che si concluderà come dianzi $p=m z-1$.

Trovato un numcro primo $p$ della forma $m z+1$ o della forma $m z-1$, se ne troverà un altro $p^{\prime}$, se in luogo di $a$ si metterà $a^{\prime}=a p$, indi un altro $p^{\prime \prime}$ se in luogo di $a^{\prime}$ si metterà $a^{\prime \prime}=a^{\prime} p^{\prime}$, e cosi via via: laonde se ne troveranno infiniti.

Alcune proprietà delle funzioni $B_{k}$ sono state dimostrate da Lagraxas o LraEndre (*).

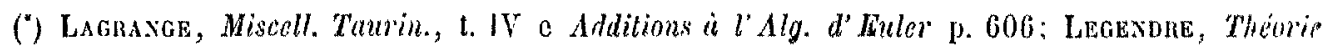
des nombres, $\mathrm{n}^{\circ}$ 143. 